> James P. Allison, né le 7 août 1948, est un immunologiste américain, récipendiaire en 2015 du Prix Albert-Lasker de la recherche médicale clinique pour la découverte et le développement d'une thérapie fondée sur l'utilisation d'un anticorps monoclonal qui permet de débloquer la réponse immunitaire pour combattre le cancer. >

\section{L'homme qui a fait sauter le verrou de la réponse immune adaptative anti-tumorale}

\author{
Jean-Luc Teillaud
}

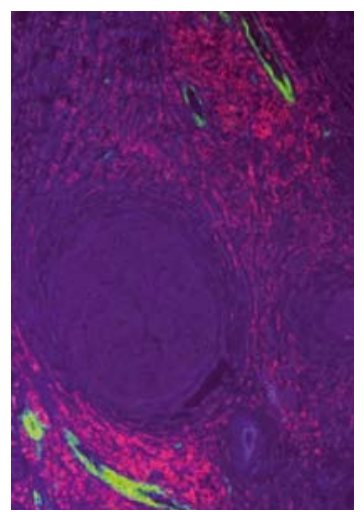

Équipe cancer et immunité antitumorale, Centre de recherche des Cordeliers, Unité mixte de recherche Inserm UMR-S 1138, Université Paris Descartes et Université Pierre et Marie Curie, 15, rue de l'École de Médecine, 75270 Paris Cedex 06, France. jean-luc.teillaud@crc.jussieu.f

apprit probablement à savoir défendre ses idées scientifiques et les démontrer sur le plan expérimental...

Des années plus tard, plongé dans des études visant à comprendre par quels mécanismes moléculaires les lymphocytes $T$ s'activent pour conduire à une réponse cellulaire puissante contre les cellules infectées par un virus ou contre des cellules tumorales, James Allison (qui était alors à l'université de Berkeley en Californie) fut parmi les tous premiers à démontrer que l'activation de ces lymphocytes T nécessitait non seulement l'engagement de leurs récepteurs pour l'antigène (TCR [T cell receptor], en charge de la reconnaissance de peptides dérivés de protéines virales ou tumorales présentés en association avec des molécules HLA du complexe majeur d'histocompatibilité (CMH), à la surface des cellules cibles) mais aussi celui d'une molécule membranaire accessoire, appelée CD28. L'analyse de la séquence de CD28 montra alors qu'une autre molécule appelée CTLA-4 (cytotoxic T-lymphocyte-associated protein-4), découverte près de dix ans auparavant en France, par Pierre Golstein [2], lui ressemblait très fortement. Cette molécule n'est exprimée que sur les lymphocytes T activés et interagit avec les mêmes molécules que CD28, exprimées à la surface des cellules présentatrices de peptides. Les premiers travaux portant sur CTLA-4 lancèrent de nombreux chercheurs sur une fausse piste, celle d'une molécule qui, comme CD28, serait responsable de l'activation T. Cependant, James Allison, et, de façon indépendante, Jeffrey Bluestone (alors à l'université de Californie à San Francisco, USCF) en collaboration avec Craig Thompson (alors au Memorial Sloan-Kettering Cancer Center à New York) firent 
l'hypothèse que l'activation cellulaire T observée lorsque CTLA-4 était ciblée pouvait s'expliquer par le blocage d'une inhibition exercée par cette molécule. C'est en 1994 et 1995 que Bluestone [3], Allison [4] et leurs collègues ont montré que CTLA-4 bloquait en fait l'activation des lymphocytes T. Bien évidemment, cette idée que CD28 et CTLA-4 conduisaient à des signaux opposés dans les lymphocytes $T$ fut d'abord très controversée, jusqu'à l'établissement de souris n'exprimant plus CTLA-4 : ces souris subissent une très forte lymphoprolifération $T$ et meurent au bout de quelques semaines.

Cette démonstration que CTLA-4 était un puissant inhibiteur de la réponse T donna alors à James Allison l'idée que les essais de vaccination anti-tumorale, alors en cours dans différents laboratoires mais décevants quant à leurs résultats cliniques, étaient limités par l'activité inhibitrice de CTLA-4. Avec sa post-doc Dana Leach, il généra alors un anticorps anti-CTLA-4 antagoniste qu'il injecta à des souris porteuses de tumeurs. Les animaux rejetèrent les tumeurs, alors que les animaux traités par un anticorps anti-CD28, ou par un anticorps non-pertinent, virent leurs tumeurs se développer et moururent [1]. Ainsi, la première démonstration expérimentale du fait que le blocage de CTLA-4 conduit à une réponse immunitaire anti-tumorale puissante était faite. Cette réponse, caractérisée par l'apparition de cellules mémoires, est capable de bloquer la progression de tumeurs établies. Mais il y a loin entre la démonstration par un scientifique (J. Allison est titulaire d'un PhD obtenu à l'université du Texas à Austin) d'un mécanisme conduisant à l'émergence d'une réponse immune adaptative anti-tumorale et le développement d'un anticorps pour un usage thérapeutique. Après avoir vainement frappé à différentes portes de l'industrie pharmaceutique, il réussit à intéresser un collègue et ami, Alan Korman, qui, avec l'aide de Nils Lonberg (ils étaients alors tous deux membres de la firme américaine Medarex), généra un anticorps humain dans des souris humanisées, appelé ipilimumab, capable de lier et d'inactiver CTLA-4. Mais les chemins de la recherche ne sont pas linéaires : les premiers essais cliniques furent jugés peu probants, la mise en place d'une immunité anti-tumorale étant marquée par une cinétique de réduction des masses tumorales très lente par rapport à celle observée quand des molécules de chimiothérapie sont utilisées, ces dernières étant directement cytotoxiques et permettant des réductions tumorales rapides. De plus, des effets secondaires sérieux furent observés. Cependant, chercheurs et cliniciens continuèrent leurs efforts pour aboutir à une meilleure utilisation de l'ipilimumab, et le premier essai de phase III publié en 2010 par Hodi (Dana-Farber Cancer Institute) et ses collègues de différents pays montra des résultats spectaculaires chez des patients atteints de mélanome métastatique [5], ce qui conduisit en
2011 la Food and drug administration (FDA) américaine à autoriser l'utilisation de l'ipilimumab chez des patients présentant des mélanomes métastatiques.

Ce travail remarquable de J. Allison, allant de la recherche fondamentale en immunologie jusqu'au développement d'un anticorps à usage thérapeutique, a stimulé un immense champ de recherche allant de l'étude du microenvironnement tumoral $(\rightarrow$ ) au développement d'anticorps antagonistes dirigés contre différentes molécules inhibitrices de la réponse T (appelées les « immune checkpoints », comme PD-1 [programmed cell death 1], $(\rightarrow)$ Voir le numéro thématique tumoraux, $m / s n^{\circ} 4$, vol. 30, avril 2014 Microenvironnements

LAG-3 [lymphocyte-activation protein 3], TIM-3 [T-cell immunoglobulin domain and mucin domain-3], VISTA [ $\mathrm{V}$ domain-containing Ig suppressor of T-cell activation], etc.), de la phagocytose par les macrophages (CD47), voire d'anticorps agonistes activant les molécules activatrices (CD28, 0X-40, CD137, etc.), pour un éventuel usage combiné de plusieurs de ces anticorps. Enfin, James Allison est un joueur de rock et de blues où il excelle dans le groupe appelé... The Checkpoints, qui réunit des immunologistes et des oncologistes de tout premier plan et où il joue de... I'harmonica. II n'est pas texan pour rien! $\diamond$

The man who blew-up the lock of the anti-tumor adaptive immune response

\section{LIENS D’INTÉRÊT}

L'auteur déclare n'avoir aucun lien d'intérêt concernant les données publiées dans cet article.

\section{RÉFÉRENCES}

1. Leach DR, Krummel MF, Allison JP. Enhancement of antitumor immunity by CTLA-4 blockade. Science 1996; 271 : 1734-6.

2. Brunet JF, Denizot F, Luciani MF, et al. A new member of the immunoglobulin superfamily, CTLA-4. Nature $1987 ; 328: 267-70$.

3. Walunas TL, Lenschow DJ, Bakker CY, et al. CTLA-4 can function as a negative regulator of T cell activation. Immunity $1994 ; 1$ : 405-13.

4. Krummel MF, Allison JP. CD28 and CTLA-4 have opposing effects on the response of T cells to stimulation. J Exp Med 1995 ; 182 : 459-65.

5. Hodi FS, O'Day SJ, McDermott DF, et al. Improved survival with ipilimumab in patients with metastatic melanoma. N Engl J Med 2010 ; 363 : 711-23.

\section{TIRÉS À PART}

J.L. Teillaud

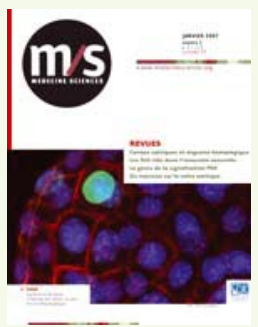

